\title{
Strain Energy Release and Intrinsic Barriers in Internal Nucleophilic Reactions
}

\author{
Joel L. Wolk, Esther Rozental, Harold Basch and Shmaryahu Hoz ${ }^{*}$
}

Department of Chemistry, Bar-Ilan University, Ramat-Gan, Israel 52900.shoz@mail.biu.ac.il

\section{Contents}

Page 1 - Figure S1

Page 1-16 - The structures and coordinates of the GS; TS and products are given.

Pages 1-6 - data for open chain compounds C; N; P; O; S.

Pages 7-16 - data for the cyclic molecules according to the order C; N; P; O; S. with the three membered rings first followed by the four membered rings.

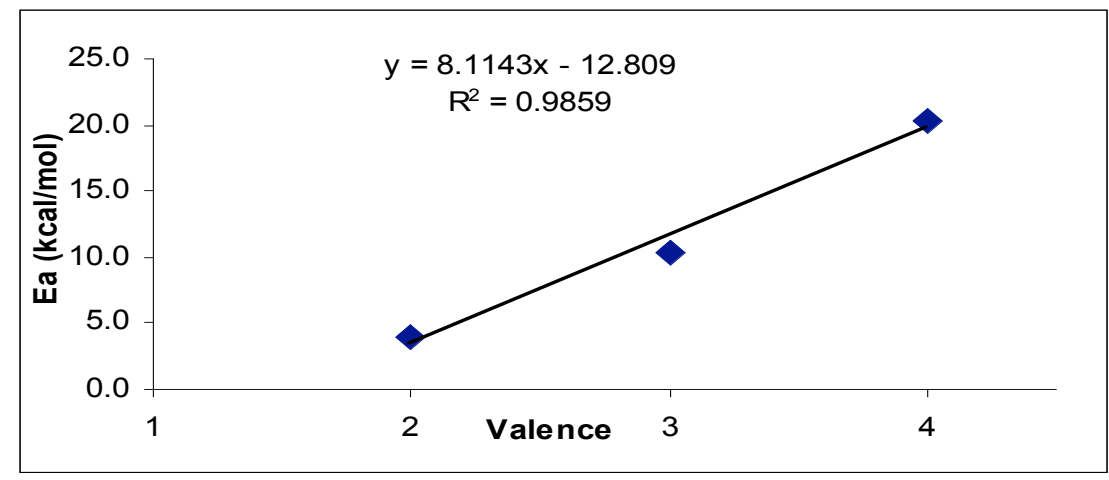

Figure S1: Intrinsic barriers as a function of the valence of $\mathbf{X}$.

$\mathrm{HF} / 6-31+\mathrm{G}^{*}$

$-\mathrm{CH} 2 \mathrm{CH} 2 \mathrm{CH} 2 \mathrm{CH} 2 \mathrm{CH} 2$

GS
E

\author{
$-195.640934$
}

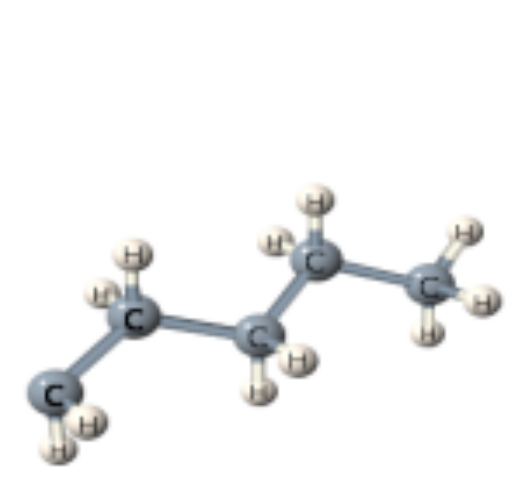

C

C

C

$\mathrm{C}$
$\mathrm{C}$
$\mathrm{C}$
$\mathrm{C}$
$\mathrm{C}$
$\mathrm{H}$
$\mathrm{H}$
$\mathrm{H}$
$\mathrm{H}$
$\mathrm{H}$
$\mathrm{H}$
$\mathrm{H}$
$\mathrm{H}$
$\mathrm{H}$
$\mathrm{H}$
$\mathrm{H}$

$\mathrm{H}$

$\mathrm{H}$

$\mathrm{H}$

$\mathrm{H}$

$\mathrm{H}$

$\mathrm{H}$

$\mathrm{H}$

$\mathrm{H}$

$\mathrm{H}$

$\mathrm{H}$

$X$
1.5276
2.26648
3.79607
4.51149
-0.37693
-0.37692
1.89422
1.89423
1.94621
1.94622
4.11744
4.11744
5.59549
4.24038
4.24037

Y

Z

$\begin{array}{rr}0 & 0 \\ 0 & 0 \\ 1.36166 & 0 \\ 1.25481 & 0 \\ 2.60848 & -0.00001 \\ 0.53136 & -0.88657 \\ 0.53135 & 0.88658 \\ -0.55673 & 0.86947 \\ -0.55673 & -0.86947 \\ 1.93447 & -0.87136 \\ 1.93447 & 0.87136 \\ 0.68275 & -0.87044 \\ 0.68276 & 0.87044 \\ 2.49658 & -0.00001 \\ 3.19338 & 0.87635 \\ 3.19337 & -0.87636\end{array}$


TS

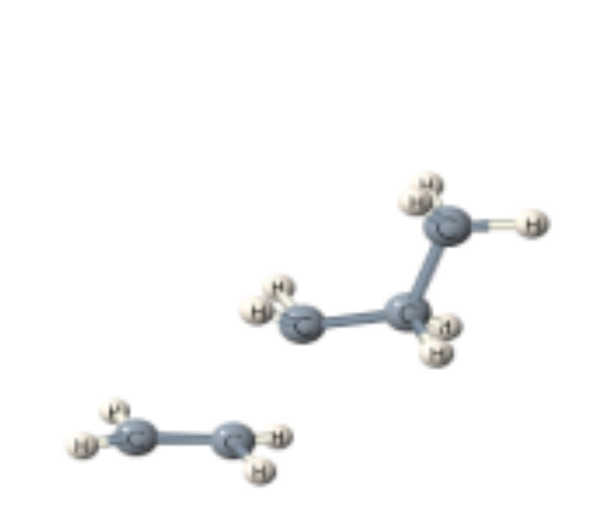

bond formed $\AA$ 1.368

bond broken $\AA$ 2.354

Product
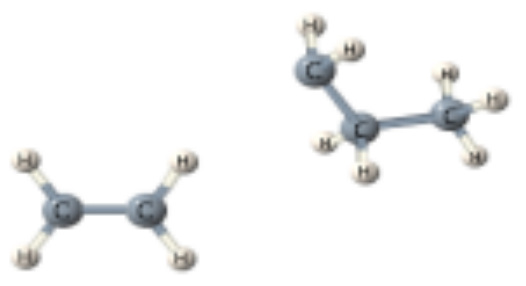

$\mathrm{MeNHCH} 2 \mathrm{NH}$

GS

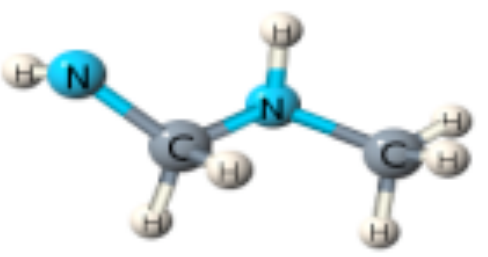

$-195.588956$

$\begin{array}{rrrr}\text { C } & 0 & 0 & 0 \\ \mathrm{C} & 1.36826 & 0 & 0 \\ \mathrm{C} & 2.38569 & 2.12229 & 0 \\ \mathrm{C} & 3.88844 & 2.35862 & -0.00113 \\ \mathrm{C} & 4.39288 & 3.81937 & -0.0014 \\ \mathrm{H} & -0.55356 & 0.1423 & -0.91536 \\ \mathrm{H} & -0.55355 & 0.14281 & 0.91528 \\ \mathrm{H} & 1.90614 & -0.21549 & 0.90205 \\ \mathrm{H} & 1.90608 & -0.21585 & -0.90202 \\ \mathrm{H} & 1.90587 & 2.54674 & -0.88933 \\ \mathrm{H} & 1.90725 & 2.54678 & 0.89007 \\ \mathrm{H} & 4.33553 & 1.86555 & -0.8716 \\ \mathrm{H} & 4.33691 & 1.86536 & 0.86853 \\ \mathrm{H} & 5.48759 & 3.88417 & -0.0022 \\ \mathrm{H} & 4.0264 & 4.34977 & 0.87516 \\ \mathrm{H} & 4.0251 & 4.34989 & -0.87734\end{array}$

$-195.612076$

$\begin{array}{rrrr}\mathrm{C} & 0 & 0 & 0 \\ \mathrm{C} & 1.32434 & 0 & 0 \\ \mathrm{C} & 4.01534 & 2.708 & 0 \\ \mathrm{C} & 4.78654 & 1.58601 & -0.68904 \\ \mathrm{C} & 6.24567 & 1.8623 & -1.12749 \\ \mathrm{H} & -0.55777 & 0.91835 & 0.07714 \\ \mathrm{H} & -0.57727 & -0.90814 & -0.07677 \\ \mathrm{H} & 1.86859 & -0.92814 & -0.07892 \\ \mathrm{H} & 1.91418 & 0.90345 & 0.0766 \\ \mathrm{H} & 3.96565 & 3.58758 & -0.65937 \\ \mathrm{H} & 4.54264 & 3.01805 & 0.91475 \\ \mathrm{H} & 4.246 & 1.26019 & -1.58478 \\ \mathrm{H} & 4.81669 & 0.70362 & -0.03943 \\ \mathrm{H} & 6.71218 & 0.99516 & -1.61252 \\ \mathrm{H} & 6.85741 & 2.13436 & -0.26869 \\ \mathrm{H} & 6.28265 & 2.69639 & -1.82653\end{array}$

$-188.615239$

$\begin{array}{rrrr}\mathrm{N} & 0 & 0 & 0 \\ \mathrm{C} & 1.44107 & 0 & 0 \\ \mathrm{H} & -0.35696 & 0.93704 & 0 \\ \mathrm{H} & 1.8092 & -1.02371 & -0.04173 \\ \mathrm{H} & 1.89036 & 0.4644 & 0.88912 \\ \mathrm{H} & 1.82857 & 0.51793 & -0.87679 \\ \mathrm{C} & -0.61393 & -0.63898 & 1.21302 \\ \mathrm{~N} & -1.93389 & -0.23406 & 1.48021 \\ \mathrm{H} & 0.01904 & -0.37302 & 2.07348 \\ \mathrm{H} & -0.45418 & -1.72265 & 1.04678 \\ \mathrm{H} & -2.52885 & -0.66601 & 0.7843\end{array}$


TS

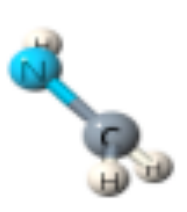

bond formed $\AA$ 1.287

Product
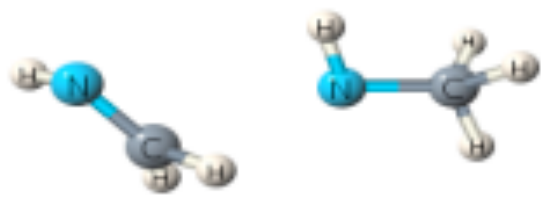

$-188.585999$

$\begin{array}{rrrr}\mathrm{N} & 0 & 0 & 0 \\ \mathrm{C} & 1.43178 & 0 & 0 \\ \mathrm{H} & -0.31359 & 0.96016 & 0 \\ \mathrm{H} & 1.80086 & -1.03129 & 0.01975 \\ \mathrm{H} & 1.91078 & 0.49917 & 0.87315 \\ \mathrm{H} & 1.91282 & 0.46069 & -0.88252 \\ \mathrm{C} & -0.79462 & -0.54787 & 2.0182 \\ \mathrm{~N} & -1.80564 & 0.1281 & 2.44048 \\ \mathrm{H} & 0.1776 & -0.2981 & 2.40866 \\ \mathrm{H} & -0.87695 & -1.52436 & 1.57075 \\ \mathrm{H} & -2.65604 & -0.26847 & 2.07619\end{array}$

$-188.588878$

$\mathrm{N}$

C

$\mathrm{H}$

$\mathrm{H}$

$\mathrm{H}$

$\mathrm{H}$

C

$\mathrm{N}$

$\mathrm{H}$

$\mathrm{H}$

$\mathrm{H}$

$\begin{array}{rrr}0 & 0 & 0 \\ 1.4333 & 0 & 0 \\ -0.30178 & 0.96714 & 0 \\ 1.80068 & -1.03244 & 0.0085 \\ 1.92605 & 0.48422 & 0.87392 \\ 1.92738 & 0.4684 & -0.88134 \\ -2.61703 & -0.54853 & 1.02581 \\ -3.47502 & 0.36741 & 0.89839 \\ -3.90381 & 0.32065 & -0.01098 \\ -2.07021 & -0.63286 & 1.94738 \\ -2.3476 & -1.25047 & 0.25492\end{array}$

$-761.221388$ 
TS

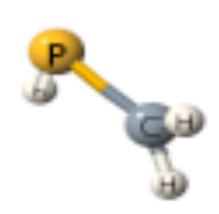

bond formed $\AA$ 1.706

Product

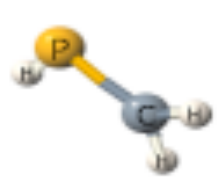

$-761.168704$

$\begin{array}{rrrr}\mathrm{P} & 0 & 0 & 0 \\ \mathrm{C} & 1.89607 & 0 & 0 \\ \mathrm{H} & -0.15639 & 1.41092 & 0 \\ \mathrm{H} & 2.23866 & -1.03107 & -0.07061 \\ \mathrm{H} & 2.33099 & 0.41963 & 0.90996 \\ \mathrm{H} & 2.32574 & 0.53713 & -0.84343 \\ \mathrm{C} & -0.29838 & 0.14991 & 2.88042 \\ \mathrm{P} & -1.67296 & 0.8416 & 3.61632 \\ \mathrm{H} & 0.57015 & 0.75742 & 2.71975 \\ \mathrm{H} & -0.13052 & -0.90417 & 2.8101 \\ \mathrm{H} & -2.43676 & -0.34033 & 3.76425\end{array}$

$-761.171987$

$\begin{array}{rrrr}\mathrm{P} & 0 & 0 & 0 \\ \mathrm{C} & 1.90601 & 0 & 0 \\ \mathrm{H} & -0.15314 & 1.41796 & 0 \\ \mathrm{H} & 2.23952 & -1.03732 & -0.01336 \\ \mathrm{H} & 2.34488 & 0.46284 & 0.8845 \\ \mathrm{H} & 2.34074 & 0.48396 & -0.87388 \\ \mathrm{C} & -0.42054 & 0.21128 & 4.05973 \\ \mathrm{P} & -1.75771 & 0.88415 & 4.7756 \\ \mathrm{H} & 0.40339 & 0.83588 & 3.76679 \\ \mathrm{H} & -0.32236 & -0.82184 & 3.78559 \\ \mathrm{H} & -2.53576 & -0.28317 & 4.9324\end{array}$

\section{$\mathrm{O}-\mathrm{CH} 2-\mathrm{OMe}$}

GS

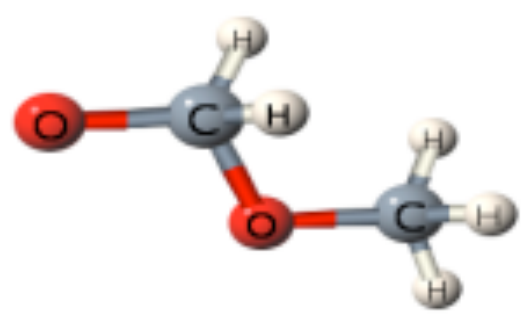

$\mathrm{O}$
$\mathrm{C}$
$\mathrm{C}$
$\mathrm{H}$
$\mathrm{H}$
$\mathrm{H}$
$\mathrm{O}$
$\mathrm{H}$
$\mathrm{H}$

$\begin{array}{rrr}0 & 0 & 0 \\ 1.3698 & 0 & 0 \\ -0.59733 & 1.36437 & 0 \\ 1.7251 & -1.0293 & 0 \\ 1.79087 & 0.49796 & 0.88163 \\ 1.79087 & 0.49796 & -0.88163 \\ -1.88053 & 1.36079 & 0 \\ -0.11695 & 1.83435 & -0.89197 \\ -0.11695 & 1.83435 & 0.89197\end{array}$


TS
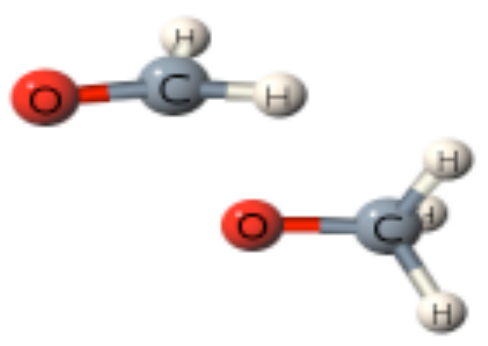

bond formed $\AA \quad$ bond broken $\AA$ 1.218 2.107

Product
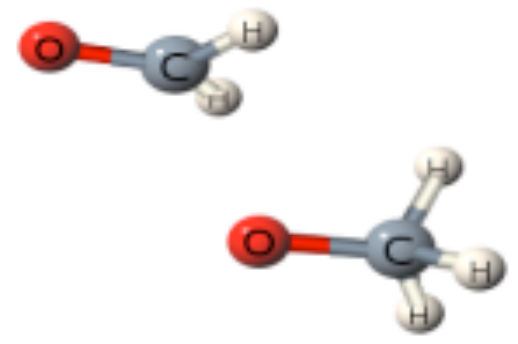

$\mathrm{MeSCH} 2 \mathrm{~S}$

GS

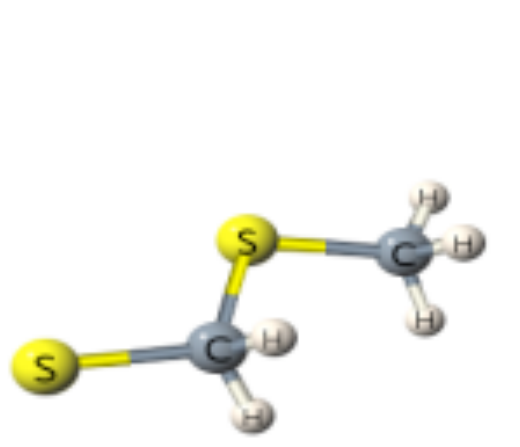

$-228.306003$

$\begin{array}{rrrr}\mathrm{O} & 0 & 0 & 0 \\ \mathrm{C} & 1.34564 & 0 & 0 \\ \mathrm{C} & -0.74031 & 1.9728 & 0 \\ \mathrm{H} & 1.80246 & -0.49617 & 0.88589 \\ \mathrm{H} & 1.8009 & 1.02647 & -0.0046 \\ \mathrm{H} & 1.80386 & -0.50298 & -0.88099 \\ \mathrm{O} & -1.81736 & 2.14429 & 0.54195 \\ \mathrm{H} & -0.66033 & 1.89445 & -1.0867 \\ \mathrm{H} & 0.1955 & 2.21123 & 0.51102\end{array}$

$-228.306049$

O

$\begin{array}{rrr}0 & 0 & 0 \\ 1.33732 & 0 & 0 \\ -0.77654 & 2.11072 & 0 \\ 1.79861 & -0.4971 & 0.88028 \\ 1.79612 & 1.0248 & -0.00002 \\ 1.79861 & -0.49713 & -0.88027 \\ -1.97452 & 2.23836 & 0.00002 \\ -0.19912 & 2.15388 & -0.91757 \\ -0.19909 & 2.15389 & 0.91755\end{array}$

$-873.680092$

$\begin{array}{rrrr}\mathrm{C} & 0 & 0 & 0 \\ \mathrm{~S} & 1.8141 & 0 & 0 \\ \mathrm{H} & -0.34214 & 1.03004 & 0 \\ \mathrm{H} & -0.39888 & -0.49197 & -0.88188 \\ \mathrm{H} & -0.39888 & -0.49197 & 0.88188 \\ \mathrm{C} & 2.13494 & -1.81143 & 0.00008 \\ \mathrm{~S} & 3.89969 & -2.22879 & 0.00004 \\ \mathrm{H} & 1.63458 & -2.20764 & -0.87704 \\ \mathrm{H} & 1.63468 & -2.20756 & 0.87729\end{array}$


TS

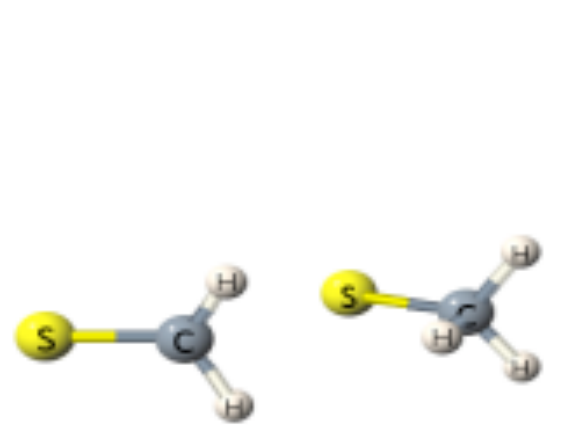

bond formed $\AA$ 1.643

Product

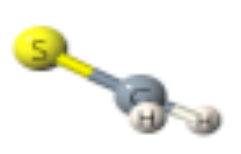

2.765
$-873.648601$

$\begin{array}{rrrr}\mathrm{C} & 0 & 0 & 0 \\ \mathrm{~S} & 1.82687 & 0 & 0 \\ \mathrm{H} & -0.40267 & 1.0099 & 0 \\ \mathrm{H} & -0.40334 & -0.503 & -0.88268 \\ \mathrm{H} & -0.40267 & -0.51478 & 0.86886 \\ \mathrm{C} & 1.94501 & -1.3677 & -2.39976 \\ \mathrm{~S} & 3.34573 & -1.79249 & -3.14503 \\ \mathrm{H} & 1.41252 & -0.48053 & -2.67717 \\ \mathrm{H} & 1.41248 & -2.05851 & -1.77784\end{array}$

bond broken $\AA$

$-873.648685$

C

$\mathrm{S}$

$\mathrm{H}$

$\mathrm{H}$

$\mathrm{H}$

C

$\mathrm{S}$

$\mathrm{H}$

$\begin{array}{rrr}0 & 0 & 0 \\ 1.82962 & 0 & 0 \\ -0.403 & 1.01029 & 0 \\ -0.40766 & -0.50353 & -0.88044 \\ -0.40301 & -0.51228 & 0.87078 \\ 1.95227 & -1.47501 & -2.57643 \\ 3.33931 & -1.89846 & -3.31602 \\ 1.44508 & -0.55801 & -2.80353 \\ 1.44502 & -2.13513 & -1.90068\end{array}$


-CH2-cyclopropane

GS

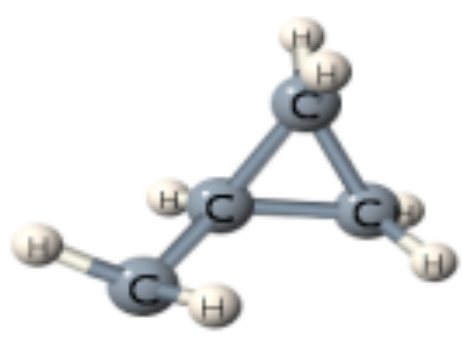

TS

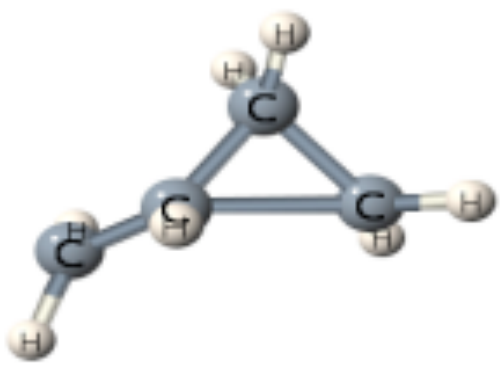

bond formed $\AA \quad$ bond broken $\AA$ 1.410

Product

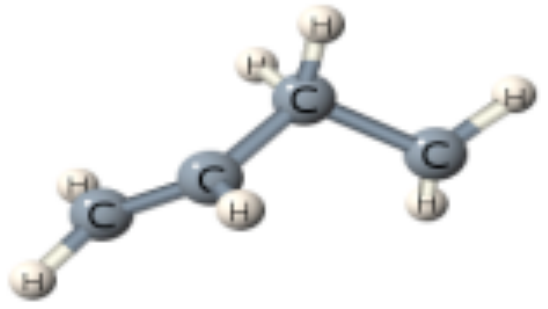

$-155.408959$

$\begin{array}{rrrr}\text { C } & 0 & 0 & 0 \\ \text { C } & 1.50775 & 0 & 0 \\ \text { C } & 2.28587 & 1.27542 & 0 \\ \text { C } & 2.40641 & 0.3493 & -1.18923 \\ \text { H } & -0.37929 & -0.91024 & -0.48331 \\ \text { H } & -0.37943 & 0.86137 & -0.56445 \\ \text { H } & 1.95493 & -0.76709 & 0.62177 \\ \text { H } & 3.14594 & 1.38493 & 0.64566 \\ \text { H } & 1.72376 & 2.18812 & -0.12415 \\ \text { H } & 3.34829 & -0.16821 & -1.3285 \\ \text { H } & 1.92043 & 0.63789 & -2.1078\end{array}$

$-155.393423$

$\begin{array}{lrrr}\mathrm{C} & 0 & 0 & 0 \\ \mathrm{C} & 1.40999 & 0 & 0 \\ \mathrm{C} & 2.2508 & 1.2008 & 0 \\ \mathrm{C} & 2.51067 & 0.70197 & -1.39763 \\ \mathrm{H} & -0.51761 & -0.89954 & -0.30688 \\ \mathrm{H} & -0.50925 & 0.91972 & -0.25936 \\ \mathrm{H} & 1.91832 & -0.90685 & 0.27364 \\ \mathrm{H} & 3.1106 & 1.1817 & 0.66367 \\ \mathrm{H} & 1.7151 & 2.13879 & 0.11672 \\ \mathrm{H} & 3.5154 & 0.38446 & -1.65192 \\ \mathrm{H} & 1.93029 & 1.1363 & -2.1965\end{array}$

$\begin{array}{rrrr}\mathrm{C} & 0 & 0 & 0 \\ \mathrm{C} & 1.34787 & 0 & 0 \\ \mathrm{C} & 2.23205 & 1.22253 & 0 \\ \mathrm{C} & 3.02245 & 1.29737 & -1.29469 \\ \mathrm{H} & -0.58214 & -0.91916 & -0.07236 \\ \mathrm{H} & -0.56491 & 0.93229 & 0.03816 \\ \mathrm{H} & 1.88562 & -0.94585 & -0.08956 \\ \mathrm{H} & 2.94142 & 1.11363 & 0.84085 \\ \mathrm{H} & 1.58052 & 2.09362 & 0.2763 \\ \mathrm{H} & 3.93974 & 1.899 & -1.21219 \\ \mathrm{H} & 2.41067 & 1.62412 & -2.14924\end{array}$


-CH2-cyclobutane

GS

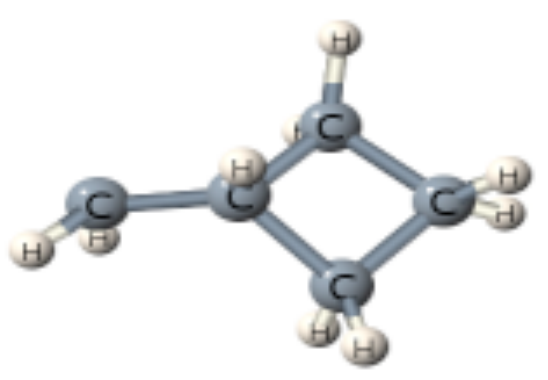

TS

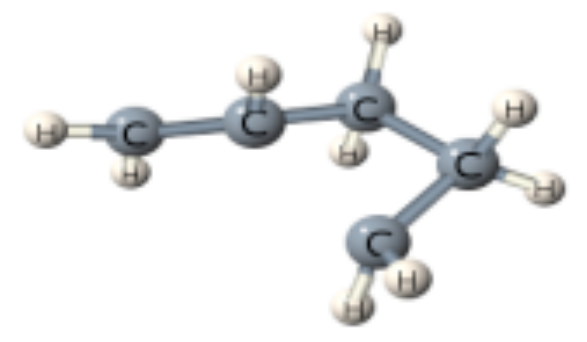

bond formed $\AA$ 1.390

bond broken $\AA$ 2.112
$-194.449273$

$\begin{array}{rrrr}\mathrm{C} & 0 & 0 & 0 \\ \mathrm{C} & 1.50221 & 0 & 0 \\ \mathrm{C} & 2.26017 & 1.35568 & 0 \\ \mathrm{C} & 3.40653 & 0.71452 & -0.81322 \\ \mathrm{C} & 2.41649 & -0.3936 & -1.22825 \\ \mathrm{H} & -0.40698 & -1.00981 & 0.12277 \\ \mathrm{H} & -0.3994 & 0.43141 & -0.92701 \\ \mathrm{H} & 1.86654 & -0.58125 & 0.85165 \\ \mathrm{H} & 2.49685 & 1.80258 & 0.96377 \\ \mathrm{H} & 1.71345 & 2.08706 & -0.59092 \\ \mathrm{H} & 3.87407 & 1.30843 & -1.5982 \\ \mathrm{H} & 4.19411 & 0.33145 & -0.16608 \\ \mathrm{H} & 1.91456 & -0.15455 & -2.16291 \\ \mathrm{H} & 2.78788 & -1.41774 & -1.28628\end{array}$

$-194.425014$

C

C

C

C

C

$\mathrm{H}$

$\mathrm{H}$

$\mathrm{H}$

$\mathrm{H}$

$\mathrm{H}$

$\mathrm{H}$

$\mathrm{H}$

$\mathrm{H}$

$\mathrm{H}$
-0.2567
-0.29598

0.952437

0.336079

$-0.77657$

$-1.13854$

$-1.0127$

1.488704

1.64001

1.050843

$-0.0918$

$-0.44267$

$-1.6986$
0.449196
0.589811

0.576885

0.375659

$-0.36672$

$-1.05379$

0.864402

$-0.04091$

1.241512

1.304841

$-0.27622$

$-0.97177$

0.352051

$-1.94027$

$-1.26472$
$-1.9603$

$-0.57075$

0.275815

1.460536

0.682044

$-2.5216$

$-2.48358$

$-0.11487$

0.490078

$-0.26062$

2.031748

2.162779

0.141338

1.23068

Product

$-194.456563$

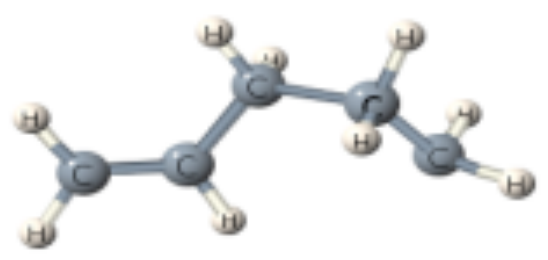

C

C

C

C

C

$\mathrm{H}$

$\mathrm{H}$

$\mathrm{H}$

$\mathrm{H}$

$\mathrm{H}$

$\mathrm{H}$

$\mathrm{H}$

$\mathrm{H}$

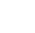

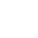

2


Amidoaziridine

GS

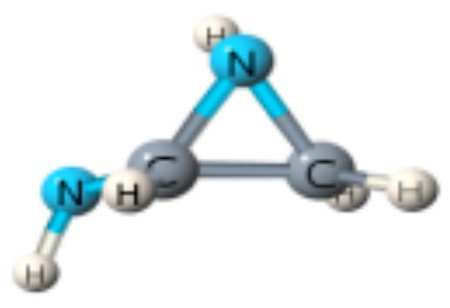

TS

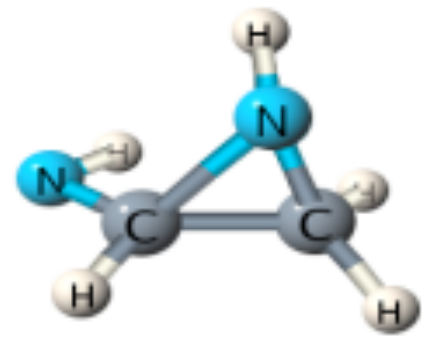

bond formed $\AA \quad$ bond broken $\AA$

1.343

1.745

Product

$-187.454047$

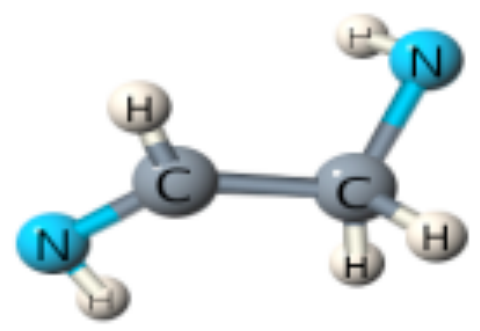

$-187.428432$

$\begin{array}{rrrr}\mathrm{C} & 0 & 0 & 0 \\ \mathrm{C} & 1.50409 & 0 & 0 \\ \mathrm{~N} & 0.734 & 1.25238 & 0 \\ \mathrm{~N} & -0.75508 & -0.28911 & -1.14035 \\ \mathrm{H} & -0.42673 & -0.19407 & 0.98802 \\ \mathrm{H} & 1.97972 & -0.2947 & -0.92225 \\ \mathrm{H} & 2.09991 & -0.1559 & 0.89181 \\ \mathrm{H} & 0.66905 & 1.60016 & -0.93881 \\ \mathrm{H} & -1.05963 & -1.25134 & -1.07208\end{array}$

$-187.425095$

C

$\begin{array}{rrr}0 & 0 & 0 \\ 1.45619 & 0 & 0 \\ 1.04064 & 1.40135 & 0 \\ -0.82153 & -0.32575 & -1.0107 \\ -0.45711 & 0.06722 & 0.97313 \\ 1.94101 & -0.39599 & -0.88978 \\ 1.99976 & -0.29466 & 0.89287 \\ 0.9819 & 1.70154 & -0.95758 \\ -0.26698 & -0.58484 & -1.81422\end{array}$

$\begin{array}{rrrr}\mathrm{C} & 0 & 0 & 0 \\ \mathrm{C} & 1.51946 & 0 & 0 \\ \mathrm{~N} & 2.09419 & 1.30807 & 0 \\ \mathrm{~N} & -0.79225 & -0.54348 & -0.82614 \\ \mathrm{H} & -0.44521 & 0.59088 & 0.79194 \\ \mathrm{H} & 1.81912 & -0.67984 & -0.82798 \\ \mathrm{H} & 1.82238 & -0.51594 & 0.91843 \\ \mathrm{H} & 1.86357 & 1.73085 & -0.89161 \\ \mathrm{H} & -0.25899 & -1.04617 & -1.52051\end{array}$


Amidoazetidine

GS

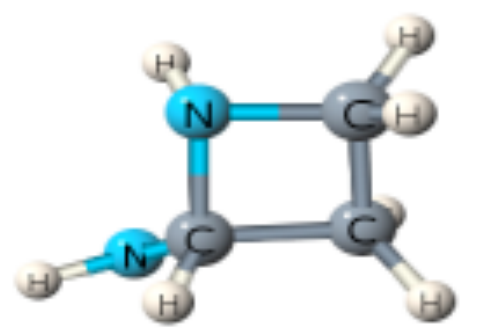

TS

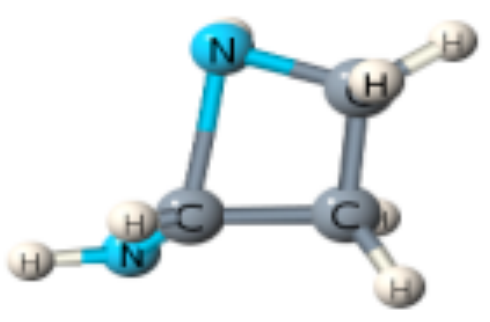

bond formed $\AA$ bond broken $\AA$ 1.315 1.950
$-226.46888$

$\begin{array}{rrrr}\mathrm{C} & 0 & 0 & 0 \\ \mathrm{C} & 1.54996 & 0 & 0 \\ \mathrm{C} & 1.50408 & 1.53545 & 0 \\ \mathrm{~N} & 0.04824 & 1.53875 & -0.21315 \\ \mathrm{~N} & -0.60732 & -0.7916 & -0.96635 \\ \mathrm{H} & -0.38349 & -0.12372 & 1.02311 \\ \mathrm{H} & 1.91205 & -0.41876 & -0.93322 \\ \mathrm{H} & 2.05274 & -0.48964 & 0.83448 \\ \mathrm{H} & 1.76971 & 1.98771 & 0.95517 \\ \mathrm{H} & 2.08766 & 2.03921 & -0.77103 \\ \mathrm{H} & -0.15817 & 1.66553 & -1.18745 \\ \mathrm{H} & -1.57869 & -0.91184 & -0.71595\end{array}$

$-226.459486$

C

C

C

$\mathrm{N}$

$\mathrm{N}$

$\mathrm{H}$

$\mathrm{H}$

$\mathrm{H}$

$\mathrm{H}$

$\mathrm{H}$

$\mathrm{H}$

$\mathrm{H}$

$\begin{array}{rrr}0 & 0 & 0 \\ 1.52153 & 0 & 0 \\ 1.65671 & 1.5225 & 0 \\ 0.3374 & 1.84881 & -0.5194 \\ -0.65535 & -0.82839 & -0.78374 \\ -0.42714 & 0.29872 & 0.9532 \\ 1.85867 & -0.43564 & -0.93678 \\ 1.97296 & -0.56482 & 0.82262 \\ 1.76003 & 1.90756 & 1.01692 \\ 2.51788 & 1.90411 & -0.55989 \\ 0.36536 & 1.74422 & -1.52267 \\ -1.63341 & -0.81761 & -0.54245\end{array}$

Product

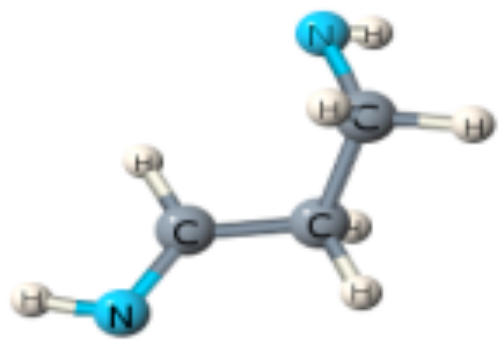

$\begin{array}{rrrr}\mathrm{C} & 0 & 0 & 0 \\ \mathrm{C} & 1.49303 & 0 & 0 \\ \mathrm{C} & 2.05821 & 1.46058 & 0 \\ \mathrm{~N} & 1.66392 & 2.27273 & -1.10316 \\ \mathrm{~N} & -0.70683 & -0.78913 & 0.69546 \\ \mathrm{H} & -0.43251 & 0.75872 & -0.63905 \\ \mathrm{H} & 1.82944 & -0.47967 & -0.9204 \\ \mathrm{H} & 1.85016 & -0.59698 & 0.8406 \\ \mathrm{H} & 1.70675 & 1.92046 & 0.93151 \\ \mathrm{H} & 3.1506 & 1.33849 & 0.14272 \\ \mathrm{H} & 2.21607 & 1.98363 & -1.90259 \\ \mathrm{H} & -1.68443 & -0.59554 & 0.55321\end{array}$


Phosphidophosphiridine

GS

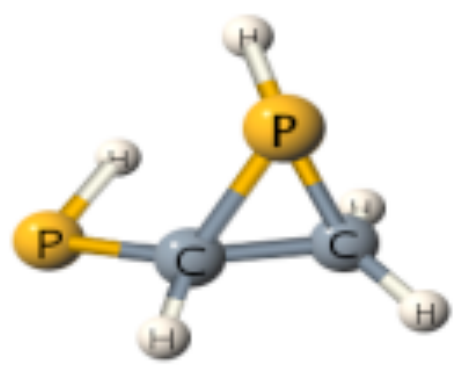

TS

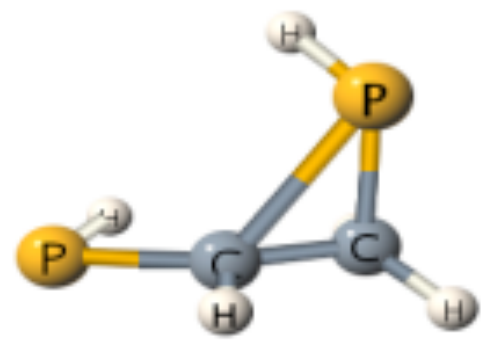

bond formed $\AA$ 1.789

bond broken $\AA$ 2.228

Product

$-760.03488$

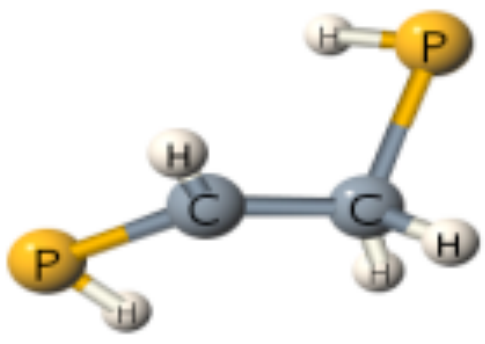

$-760.024935$

$\begin{array}{rrrr}\mathrm{C} & 0 & 0 & 0 \\ \mathrm{C} & 1.45345 & 0 & 0 \\ \mathrm{P} & 1.2005 & 1.87722 & 0 \\ \mathrm{P} & -1.12994 & -0.53808 & -1.27864 \\ \mathrm{H} & -0.44641 & 0.05339 & 0.97746 \\ \mathrm{H} & 1.93159 & -0.41234 & -0.8744 \\ \mathrm{H} & 1.93144 & -0.32811 & 0.91367 \\ \mathrm{H} & 1.08273 & 2.00813 & -1.3962 \\ \mathrm{H} & -0.15361 & -0.7165 & -2.29415\end{array}$

$\begin{array}{rrrr}\text { C } & 0 & 0 & 0 \\ \mathrm{C} & 1.48413 & 0 & 0 \\ \mathrm{P} & 0.78187 & 1.72983 & 0 \\ \mathrm{P} & -1.08145 & -0.75839 & -1.32506 \\ \mathrm{H} & -0.42379 & -0.18079 & 0.97814 \\ \mathrm{H} & 1.96909 & -0.33356 & -0.90178 \\ \mathrm{H} & 2.00664 & -0.29007 & 0.90067 \\ \mathrm{H} & 0.79229 & 1.93419 & -1.38961 \\ \mathrm{H} & -0.22051 & -0.42732 & -2.40886\end{array}$

0

0

$-760.029389$ 
Phosphidophosphetidine

GS

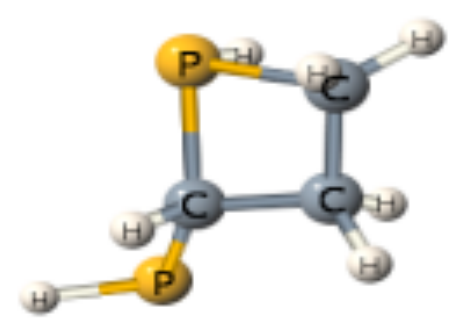

TS

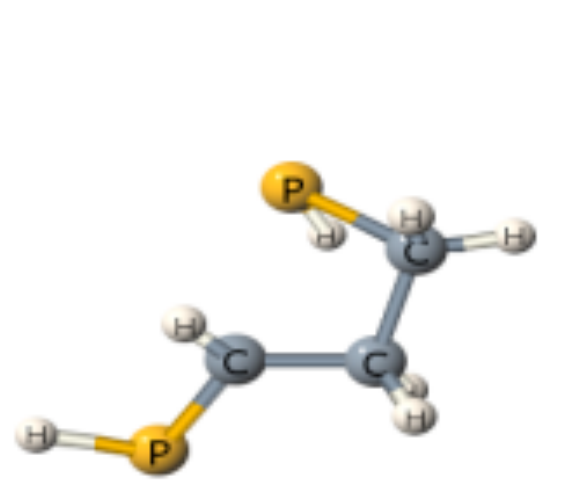

bond formed $\AA \quad$ bond broken $\AA$ 1.745 2.537

Product
$-799.066658$

$\begin{array}{rrrr}\text { C } & 0 & 0 & 0 \\ \mathrm{C} & 1.5482 & 0 & 0 \\ \mathrm{C} & 1.76738 & 1.52953 & 0 \\ \mathrm{P} & 0.02196 & 1.77603 & -0.65777 \\ \mathrm{P} & -0.83721 & -1.44325 & -0.87624 \\ \mathrm{H} & -0.35293 & 0.07514 & 1.0261 \\ \mathrm{H} & 1.91084 & -0.42877 & -0.93047 \\ \mathrm{H} & 2.00723 & -0.55231 & 0.81896 \\ \mathrm{H} & 1.84903 & 1.93191 & 1.00679 \\ \mathrm{H} & 2.6004 & 1.90751 & -0.58606 \\ \mathrm{H} & 0.3113 & 1.47718 & -2.00171 \\ \mathrm{H} & -2.07928 & -1.34262 & -0.18929\end{array}$

$-799.043987$

C

C

C

$P$

$P$

$\mathrm{H}$

$\mathrm{H}$

$\mathrm{H}$

$\mathrm{H}$

$\mathrm{H}$

$\mathrm{H}$

$\mathrm{H}$
$-799.06069$

$\begin{array}{rrrr}\mathrm{C} & 0 & 0 & 0 \\ \mathrm{C} & 1.49757 & 0 & 0 \\ \mathrm{C} & 2.09668 & 1.42873 & 0 \\ \mathrm{P} & 1.81168 & 2.4942 & -1.54876 \\ \mathrm{P} & -0.96054 & -0.88725 & 1.0435 \\ \mathrm{H} & -0.42965 & 0.65255 & -0.74129 \\ \mathrm{H} & 1.82641 & -0.50421 & -0.90711 \\ \mathrm{H} & 1.86685 & -0.58195 & 0.84535 \\ \mathrm{H} & 1.68485 & 1.97344 & 0.84877 \\ \mathrm{H} & 3.16095 & 1.32506 & 0.20194 \\ \mathrm{H} & 2.69097 & 1.74697 & -2.38728 \\ \mathrm{H} & -2.2266 & -0.47269 & 0.57136\end{array}$


Oxyoxirane

GS

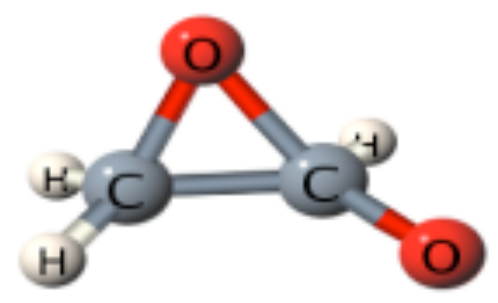

TS

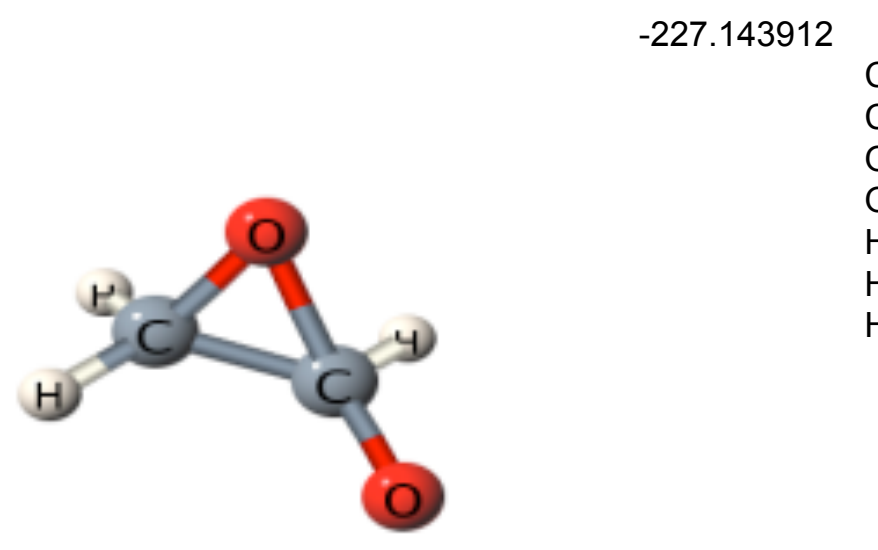

bond formed $\AA \quad$ bond broken $\AA$

1.260

1.591

Product

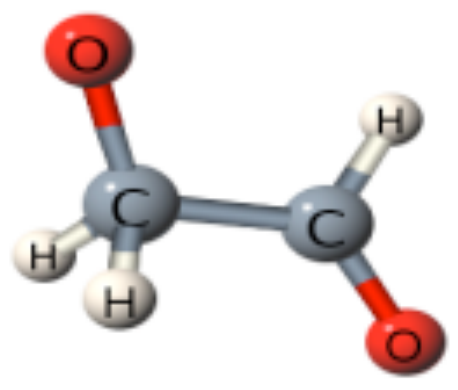

$-227.170815$

$-227.143959$

C

C

O

$\mathrm{O}$

$\mathrm{H}$

$\mathrm{H}$

$\mathrm{H}$

$\begin{array}{rrr}0 & 0 & 0 \\ 1.46649 & 0 & 0 \\ 0.86218 & 1.26812 & 0 \\ -0.74622 & -0.29599 & -0.98175 \\ -0.42985 & -0.03705 & 1.00626 \\ 1.9698 & -0.26731 & -0.92174 \\ 2.05298 & -0.23301 & 0.88746\end{array}$

C

C

$\mathrm{O}$

O

$\mathrm{H}$

$\mathrm{H}$

$\mathrm{H}$

$\begin{array}{rrr}0 & 0 & 0 \\ 1.46034 & 0 & 0 \\ 0.92745 & 1.29298 & 0 \\ -0.74716 & -0.27675 & -0.97657 \\ -0.43291 & 0.01609 & 1.00357 \\ 1.95754 & -0.2962 & -0.91815 \\ 2.03698 & -0.26743 & 0.88642\end{array}$

C

C

O

$\mathrm{O}$

$\mathrm{H}$

$\mathrm{H}$

$\mathrm{H}$

$\begin{array}{rrr}0 & 0 & 0 \\ 1.5285 & 0 & 0 \\ 2.04794 & 1.22124 & 0 \\ -0.72746 & -0.93837 & -0.19402 \\ -0.43101 & 0.98971 & 0.20885 \\ 1.80355 & -0.64487 & -0.86265 \\ 1.78347 & -0.62825 & 0.88887\end{array}$


Oxyoxetane

GS

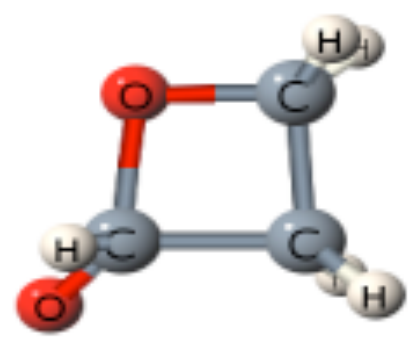

TS

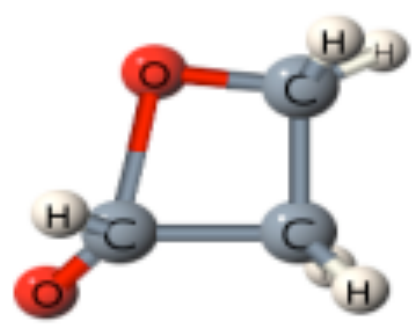

bond formed $\AA \quad$ bond broken $\AA$ 1.237 1.787

Product

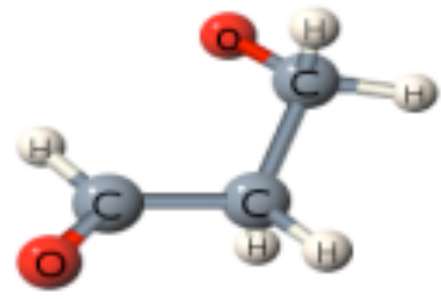

$-266.184497$

$\begin{array}{rrrr}\text { C } & 0 & 0 & 0 \\ \text { C } & 1.56239 & 0 & 0 \\ \text { C } & 1.47111 & 1.52292 & 0 \\ \text { O } & 0.10759 & 1.52447 & -0.29077 \\ \text { O } & -0.6631 & -0.70789 & -0.81547 \\ \text { H } & -0.36699 & -0.00369 & 1.04598 \\ \text { H } & 1.93474 & -0.41214 & -0.93239 \\ \text { H } & 2.0588 & -0.48161 & 0.8422 \\ \text { H } & 1.69669 & 1.97514 & 0.97211 \\ \text { H } & 2.06391 & 2.05077 & -0.74957\end{array}$

$-266.18273$

$\begin{array}{rrrr}\text { C } & 0 & 0 & 0 \\ \mathrm{C} & 1.53682 & 0 & 0 \\ \mathrm{C} & 1.57252 & 1.52619 & 0 \\ \mathrm{O} & 0.27452 & 1.71633 & -0.41476 \\ \mathrm{O} & -0.67695 & -0.68958 & -0.77165 \\ \mathrm{H} & -0.41051 & 0.21021 & 0.996 \\ \mathrm{H} & 1.8894 & -0.42827 & -0.93332 \\ \mathrm{H} & 1.99618 & -0.53166 & 0.83659 \\ \mathrm{H} & 1.77561 & 1.9311 & 1.00429 \\ \mathrm{H} & 2.32363 & 1.96869 & -0.66568\end{array}$

$-266.204491$

$\begin{array}{rrrr}\mathrm{C} & 0 & 0 & 0 \\ \mathrm{C} & 1.49343 & 0 & 0 \\ \mathrm{C} & 2.0536 & 1.4627 & 0 \\ \mathrm{O} & 1.63198 & 2.17139 & -1.03906 \\ \mathrm{O} & -0.69916 & -0.69362 & 0.69394 \\ \mathrm{H} & -0.45116 & 0.70769 & -0.69369 \\ \mathrm{H} & 1.81489 & -0.45274 & -0.93852 \\ \mathrm{H} & 1.8526 & -0.60913 & 0.82965 \\ \mathrm{H} & 1.77516 & 1.88853 & 0.9934 \\ \mathrm{H} & 3.15879 & 1.33865 & 0.06706\end{array}$


Thiaoxythiaoxirane

GS

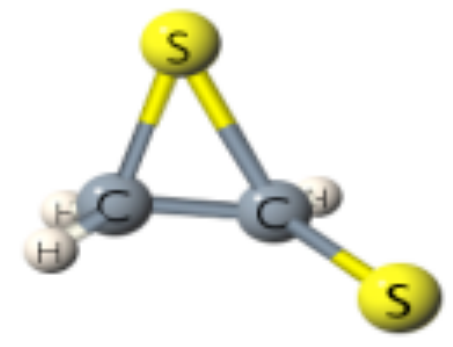

Product

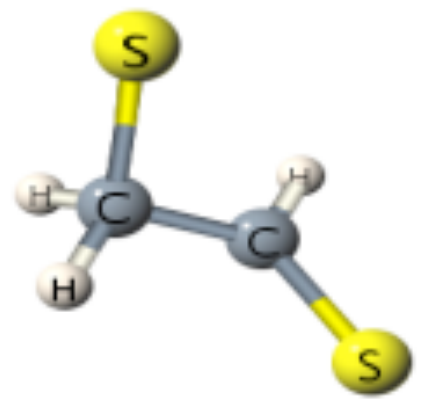

Thiaoxythiaoxetane

GS

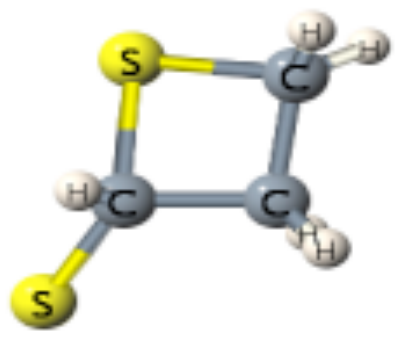

$-872.505747$

$\begin{array}{lrrr}\text { C } & 0 & 0 & 0 \\ \text { C } & 1.45504 & 0 & 0 \\ \text { S } & 0.97851 & 1.7661 & 0 \\ \text { S } & -1.0021 & -0.55456 & -1.31337 \\ H & -0.44197 & -0.02261 & 0.97946 \\ H & 1.92209 & -0.33256 & -0.91056 \\ H & 1.96601 & -0.31621 & 0.89695\end{array}$

$-872.513463$

C

$\begin{array}{rrr}0 & 0 & 0 \\ 1.47932 & 0 & 0 \\ 1.98126 & 1.77341 & 0 \\ -1.00785 & -0.59296 & -1.14449 \\ -0.43157 & 0.46667 & 0.87078 \\ 1.86053 & -0.54024 & -0.85561 \\ 1.8285 & -0.48201 & 0.91038\end{array}$

$-911.535048$

$\begin{array}{lrrr}\text { C } & 0 & 0 & 0 \\ \mathrm{C} & 1.54048 & 0 & 0 \\ \mathrm{C} & 1.73357 & 1.52469 & 0 \\ \mathrm{~S} & 0.04913 & 1.77993 & -0.66727 \\ \mathrm{~S} & -0.89437 & -1.29 & -0.8484 \\ \mathrm{H} & -0.36032 & 0.10908 & 1.01523 \\ \mathrm{H} & 1.89271 & -0.43046 & -0.92898 \\ \mathrm{H} & 1.99216 & -0.54119 & 0.82982 \\ \mathrm{H} & 1.8398 & 1.9426 & 0.99583 \\ \mathrm{H} & 2.53143 & 1.90599 & -0.62709\end{array}$


TS

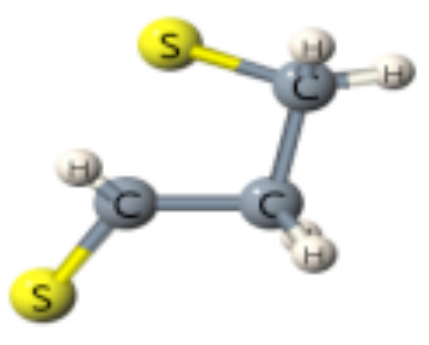

bond formed $\AA \quad$ bond broken $\AA$ 1.692

Product

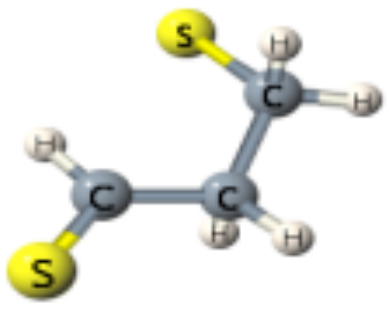

$-911.542549$

$-911.526938$

$\begin{array}{rrrr}\mathrm{C} & 0 & 0 & 0 \\ \mathrm{C} & 1.51805 & 0 & 0 \\ \mathrm{C} & 1.86063 & 1.48508 & 0 \\ \mathrm{~S} & 0.42829 & 2.09734 & -0.9541 \\ \mathrm{~S} & -0.93516 & -1.21372 & -0.71692 \\ \mathrm{H} & -0.42368 & 0.47865 & 0.86422 \\ \mathrm{H} & 1.86168 & -0.46927 & -0.91293 \\ \mathrm{H} & 1.90436 & -0.5764 & 0.84498 \\ \mathrm{H} & 1.86672 & 1.89342 & 1.0073 \\ \mathrm{H} & 2.81973 & 1.71009 & -0.45553\end{array}$

C

C

$\begin{array}{rrr}0 & 0 & 0 \\ 1.4909 & 0 & 0 \\ 2.0882 & 1.42447 & 0 \\ 1.70326 & 2.40535 & -1.4925 \\ -0.97403 & -0.93922 & 0.9029 \\ -0.43251 & 0.7116 & -0.68399 \\ 1.79845 & -0.48473 & -0.92593 \\ 1.85392 & -0.5956 & 0.83359 \\ 1.74901 & 1.93671 & 0.89846 \\ 3.16344 & 1.30538 & 0.10487\end{array}$

